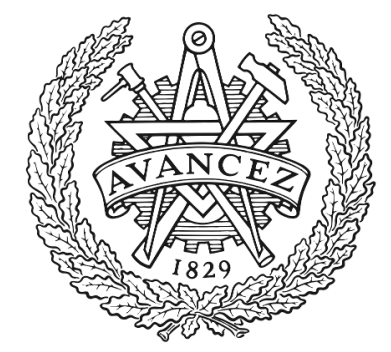

CHALMERS

UNIVERSITY OF TECHNOLOGY

\title{
Design, fabrication, and characterization of a highly nonlinear few-mode fiber
}

Downloaded from: https://research.chalmers.se, 2023-04-26 15:13 UTC

Citation for the original published paper (version of record):

Gao, J., Nazemosadat Arsanjani, S., Yang, C. et al (2019). Design, fabrication, and characterization of a highly nonlinear few-mode fiber. Photonics Research, 7(11): 1354-1362.

http://dx.doi.org/10.1364/PRJ.7.001354

N.B. When citing this work, cite the original published paper. 


\title{
Design, fabrication and characterization of highly nonlinear few-mode fiber
}

\author{
Jitao GaO ${ }^{1,+}$, Elham Nazemosadat ${ }^{2,+}$, Chen YanG $^{3}$, Songnian Fu ${ }^{1,},{ }^{*}$, Ming Tang ${ }^{1}$, Weijun Tong ${ }^{3}$, \\ Joel Carpenter ${ }^{4}$, Jochen Schröder ${ }^{2}$, Magnus Karlsson ${ }^{2}$, and Peter A. Andrekson ${ }^{2}$
}

\author{
${ }^{1}$ Wuhan National Laboratory for Optoelectronics, and School of Optics and Electronic Information, Huazhong University of Science and Technology, Wuhan, \\ 430074, China \\ ${ }^{2}$ Photonics Laboratory, Department of Microtechnology and Nanoscience, Chalmers University of Technology, Gothenburg 41296, Sweden \\ ${ }^{3}$ State Key Laboratory of Optical Fibre and Cable Manufacture Technology, Yangtze Optical Fibre and Cable Joint Stock Limited Company (YOFC), Wuhan \\ 430073, China \\ ${ }^{4}$ School of Information Technology and Electrical Engineering, The University of Queensland, Brisbane, Qld 4072, Australia \\ ${ }^{+}$These authors contributed equally to this work. \\ *Corresponding author: songnian@mail.hust.edu.cn
}

\begin{abstract}
We present the design, fabrication and characterization of a highly-nonlinear few-mode fiber (HNL-FMF) with an inter-modal nonlinear coefficient of $2.8(\mathrm{~W} \cdot \mathrm{km})^{-1}$, which to the best of our knowledge is the highest reported to date. The graded-index circular core fiber s upports two mode groups (MGs) with 6 eigenmodes and is highly doped with germanium. This breaks the mode degeneracy within the higherorder MG, leading to different group velocities among corresponding eigenmodes. Thus, the HNL-FMF can support multiple inter-modal four-wave mixing (FWM) processes between the two MGs, at the same time. In a proof of concept experiment, we demonstrate simultaneous inter-modal wavelength conversions among three eigenmodes of the HNL-FMF over the C-band.
\end{abstract}

\section{INTRODUCTION}

Multicore and multimode fibers have gained interest in recent years, as they can potentially increase the capacity of telecommunication systems by transmitting data through individual cores or spatial modes of the fiber [1-3]. In data transmission, any nonlinear interaction among the cores of a multicore or the spatial modes of a multimode fiber should be avoided as it leads to crosstalk. However, based on the characteristics found in a multimode fiber [4], intentional nonlinear interactions in such fibers could be used for ultrafast all-optical signal processing applications such as multimode parametric amplification [5-7], mode and wavelength conversion [8-14], optical switching [15], multimode supercontinuum generation [16] and construction of high power fiber lasers [17]. Such applications have motivated researches on both intra- $[5,7]$ and inter-modal FWM processes in multimode fibers [18-22].

In an intra-modal FWM process, where all the participating waves are in the same spatial mode, to achieve phase matching over a wide bandwidth, the pump should be placed close to the zero-dispersion wavelength and in the anomalous dispersion regime $[23,24]$. Inter-modal FWM processes have a more flexible phase-matching condition, as they can be phase-matched away from the zero-dispersion wavelength, thanks to the different dispersion properties of individual spatial modes [25]. This makes inter-modal processes particularly interesting. The main inter-modal FWM processes, known as phase conjugation (PC) and Bragg scattering (BS), lead to wavelength and mode conversion. In the PC process, two pump beams amplify a signal and generate a phase conjugated idler, while in the BS process two pumps mediate an exchange of energy between the signal and idler photons [26].

To date, most studies conducted on inter-modal nonlinear processes have been carried out in commercially available fewmode fibers (FMFs) which have a relatively low inter-modal nonlinear coefficient of less than $0.9(\mathrm{~W} \cdot \mathrm{km})^{-1}$ [8]. To increase the efficiency of these nonlinear interactions, reduce power consumption, and shorten the fiber length [14], FMF with highly nonlinear coefficient would be beneficial, which can be realized by raising the germanium doping concentration and decreasing the inter-modal effective area. Recently, a dispersion engineered HNL-FMF with an inter-modal effective area of $50 \mu \mathrm{m}^{2}$ has been reported, where inter-modal wavelength conversion was demonstrated over $40 \mathrm{~nm}$ [27]. Here, we report HNL-FMF with a smaller inter-modal effective area of less than $43 \mu \mathrm{m}^{2}$, corresponding to an inter-modal nonlinear coefficient 
of $2.8(\mathrm{~W} \cdot \mathrm{km})^{-1}$, which is to the best of our knowledge the highest reported to date. In addition, the non-degenerate eigenmodes in the higher-order MG of the HNL-FMF have different group velocities, allowing many inter-modal FWM processes to occur at the same time, including interactions among the eigenmodes within one MG. In a proof of concept experiment, we demonstrate simultaneous inter-modal FWM among three eigenmodes of the HNL-FMF. Moreover, due to its specific dispersion properties, our HNL-FMF could potentially be used to achieve mode exchange, i.e., mode conversion which is not accompanied by wavelength conversion. This is a unique feature of this fiber, as in all other inter-modal interactions demonstrated so far, mode conversion and wavelength conversion always occur side-by-side [9-11].

\section{THEORY}

Let us assume that pump $p$ at frequency $\omega_{p}$ and signal $s$ at $\omega_{s}$ are launched into the HNL-FMF and coupled to the spatial mode $a$, while pump $q$ at frequency $\omega_{q}$ is coupled to mode $b$ of the HNL-FMF. Intra-modal FWM, referred to as modulational instability (MI), leads to the generation of an idler in mode $a$ at $\omega_{i, \mathrm{MI}}=2 \omega_{p}-\omega_{s}$, if the phase-matching conditions are satisfied. Assuming that both inter-modal PC and BS processes are also phase-matched, each process generates an idler in mode $b$ at $\omega_{i, P C}$ and $\omega_{i, B S}$, respectively, as shown in Fig. 1(a). The linear phase mismatch of these processes are $\Delta \beta_{\mathrm{PC}}(\omega)=\beta^{a}\left(\omega_{s}\right)+\beta^{b}\left(\omega_{i, \mathrm{PC}}\right)-\beta^{a}\left(\omega_{p}\right)-\beta^{b}\left(\omega_{q}\right)$ and $\Delta \beta_{\mathrm{BS}}(\omega)=\beta^{a}\left(\omega_{s}\right)+\beta^{b}\left(\omega_{q}\right)-\beta^{a}\left(\omega_{p}\right)-\beta^{b}\left(\omega_{i, \mathrm{BS}}\right)$, respectively. In both processes, if we expand $\beta^{a}\left(\omega_{s}\right)$ and $\beta^{a}\left(\omega_{p}\right)$ at $\left(\omega_{s}+\omega_{p}\right) / 2, \beta^{b}\left(\omega_{i}\right)$ and $\beta^{b}\left(\omega_{q}\right)$ at $\left(\omega_{i}+\omega_{q}\right) / 2$ in Taylor series, respectively, the phase mismatch can be approximated by [8]:

$$
\Delta \beta \approx\left(\omega_{s}-\omega_{p}\right)\left[\beta_{1}^{a}\left(\frac{\omega_{s}+\omega_{p}}{2}\right)-\beta_{1}^{b}\left(\frac{\omega_{i}+\omega_{q}}{2}\right)\right],
$$

where $\beta^{m}(\omega)$ and $\beta_{1}^{m}(\omega)$ represent the propagation constant and the inverse group velocity of mode $m$ at frequency $\omega$. For simplicity, the contribution of higher order dispersion terms has been ignored. Note that we do not neglect group velocity dispersion $\beta_{2}$ here, because all the even order terms of the propagation constants in the expression of $\Delta \beta$ are zero, which means the effect of $\beta_{2}$ is already included in the calculated or measured $\beta_{1}$ values at the two wavelengths. Equation 1 shows that the PC and BS processes are phase-matched $(\Delta \beta=0)$, when $\beta_{1}$ of mode $a$ at the average frequency of the signal and pump in mode $a, \omega_{A}=\left(\omega_{p}+\omega_{s}\right) / 2$, is nearly equal to $\beta_{1}$ of mode $b$ at the average frequency of the idler and pump in mode $b$, $\omega_{B}=\left(\omega_{q}+\omega_{i}\right) / 2$ [8]. In other words, as illustrated in Fig. 1(b), $\beta_{1}^{a}$ at $\omega_{A}$ should lie on the same horizontal line as $\beta_{1}^{b}$ at $\omega_{B}$, for the PC and BS processes [10]. Meanwhile, as shown in Eq. 1, the frequency detuning of the signal from the pump plays a role in the phase mismatch as well. Thus, it is not easy to maintain phase matching for signals that are far away from the pump wavelength.

\section{FIBER DESIGN AND FABRICATION}

We have designed a circularly symmetric HNL-FMF with a graded index core and a double cladding configuration [7]. The design is optimized so that the dispersion properties of the modes in the two lowest-order MGs fulfill the phase-matching conditions for inter-modal FWM over the C-band. The refractive index (RI) profile of the designed fiber and that of the fabricated

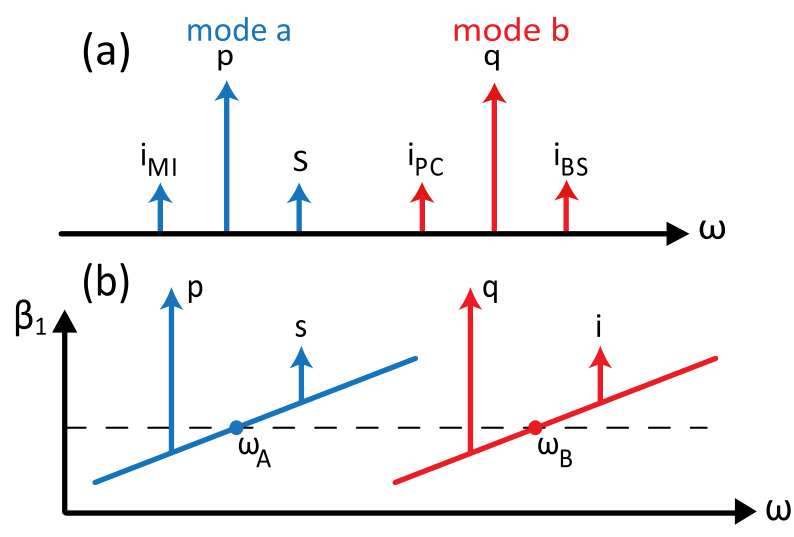

Fig. 1. (a) Schematic of the intra- and inter-modal FWM processes. The colors represent the spatial mode of the waves. (b) The inverse group velocity $\left(\beta_{1}\right)$ vs. angular frequency relation required for phase matching in the inter-modal processes. The dashed lines indicate the $\beta_{1}$ values at the average frequency of the two waves in each mode, which should be equal.

HNL-FMF are shown in Fig. 2(a), where the designed core radius is $R=4.3 \mu \mathrm{m}$ and the RI profile exponent is $\alpha=2.16$. The fiber supports two MGs, referred to as MG1 and MG2, respectively. The transverse mode profiles of the supported modes at $1550 \mathrm{~nm}$ are depicted in the inset of Fig. 2(a). The core is highly doped with germanium ( $25 \mathrm{~mol} . \%)$, thus the RI difference between the core and cladding is relatively large and consequently the degeneracy between the linearly polarized $\mathrm{LP}_{11}$ modes in MG2 is broken. Therefore, the eigenmodes have been considered in this work, where two $\mathrm{HE}_{11}$ eigenmodes belong to MG1, while $\mathrm{TE}_{01}$, $\mathrm{TM}_{01}$ and two $\mathrm{HE}_{21}$ eigenmodes belong to MG2. The effective RIs of $\mathrm{HE}_{11}, \mathrm{TE}_{01}, \mathrm{TM}_{01}$ and $\mathrm{HE}_{21}$ modes are 1.4697, 1.4572, 1.4572 and 1.4571, respectively. In MG2, the $\mathrm{TE}_{01}$ and $\mathrm{TM}_{01}$ modes have slightly different propagation constants, which is also different from that of the degenerate even and odd $\mathrm{HE}_{21}$ eigenmodes. Even such small differences in propagation constants lead to different group velocities for the modes within MG2. This can be seen in Fig. 2(b), which shows the simulated relative inverse group velocity $\left(\Delta \beta_{1}\right)$ between the supported modes. Taking the $\beta_{1}$ of $\mathrm{HE}_{21}$ mode at $1530 \mathrm{~nm}$ for a reference, the $\Delta \beta_{1}$ between $\mathrm{HE}_{21}$ mode and an arbitrary eigenmode at an arbitrary wavelength can be obtained. Even though the RI profile of the fabricated fiber is close to the design, the measured $\Delta \beta_{1}$ values do not match the simulations, which can be attributed to the inevitable variations along the fiber length during the fiber drawing. Simulations reveal that even small variations of $\pm 1 \%$ in the $R$ and $\alpha$ parameters lead to changes of as much as $\sim 200 \mathrm{ps} / \mathrm{km}$ in $\Delta \beta_{1}$ between $\mathrm{HE}_{11}$ mode in MG1 and any of the eigenmodes in MG2, as shown in Fig. 2(c). Meanwhile, $\Delta \beta_{1}$ between the eigenmodes within MG2 remains rather constant. The reason is that even though the variations in the fiber parameters affect the propagation constant $\beta$ of the modes (mainly those in MG2 since they are higher order modes), $\beta$ values within one MG change with a similar rate. Therefore, $\Delta \beta_{1}$ between any two of the eigenmodes in MG2 almost remains fixed, whereas it changes considerably between MG1 and MG2. Such high sensitivity to the deviation from the optimal parameters should be carefully considered during the fabrication process of the HNL-FMF, given that inter-modal FWM interactions rely on the $\Delta \beta_{1}$ profile of the HNL-FMF. Considering the inevitable fabri- 

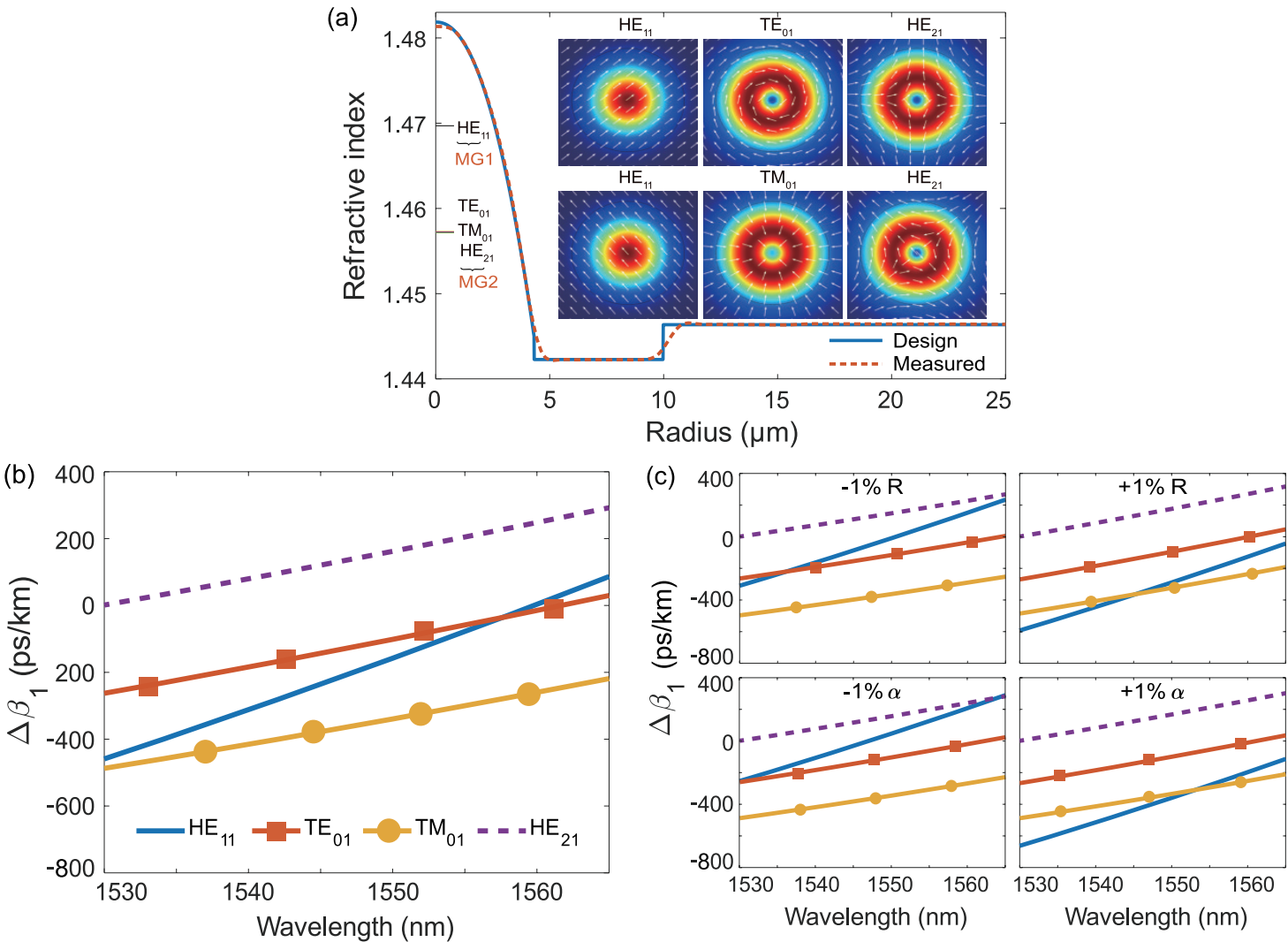

Fig. 2. (a) Designed and measured refractive index profile of the fabricated HNL-FMF at $1550 \mathrm{~nm}$. The effective refractive index of the supported modes and their transverse mode profiles are also shown. Relative inverse group velocity curves of the modes of the (b) designed fiber and (c) those of the modes when the core radius or $\alpha$ parameters are changed $\pm 1 \%$ from their optimal values.

cation imperfections and inhomogeneous stress caused micro deformation of the fiber, characterization of $\Delta \beta_{1}$ values for all guided modes is necessary for the practical HNL-FMF based applications. If we set wavelengths of pumps and signal following the designed $\Delta \beta_{1}$, the inter-modal FWM processes will very likely to be phase-mismatched.

The fiber core has a small radius and a high doping concentration, leading to a relatively high nonlinearity. The nonlinear coefficients can be calculated using $\gamma=\omega n_{2} /\left(c A_{\text {eff }}\right)$, where $c$ is the speed of light in vacuum, $n_{2}$ is the equivalent nonlinear refractive index over the core area according to the expression provided in Ref. [28], and $A_{\text {eff }}$ is the effective mode area. The effective areas of MG1 and MG2, calculated using the overlap integral of the spatial distribution of each $\mathrm{MG}$, are approximately 21.3 and $42.9 \mu \mathrm{m}^{2}$, respectively. The inter-modal $A_{\text {eff }}$ between the two MGs is around $42.5 \mu \mathrm{m}^{2}$. Accordingly, the calculated nonlinear coefficient $\gamma$ of MG1 is $6(\mathrm{~W} \cdot \mathrm{km})^{-1}$ and that of MG2 is $3(\mathrm{~W} \cdot \mathrm{km})^{-1}$, while the inter-modal nonlinear coefficient between MG1 and MG2 is $3(\mathrm{~W} \cdot \mathrm{km})^{-1}$.

As seen in Fig. 2(b), the $\beta_{1}$ curve of the mode in MG1 intersects with each curve of the modes in MG2 at a specific wavelength. Going back to Fig. 1(b), we have $\omega_{A}=\omega_{B}$ at the intersecting wavelengths. In such conditions, if the pumps in both modes are placed at the same frequency such that $\omega_{p}=\omega_{q}$, a signal at $\omega_{s}$ in mode $a$ can be converted to an idler at $\omega_{i}$ in mode $b$ or vice versa through the BS process, such that $\omega_{s}=\omega_{i}$. Thus, our HNL-FMF can potentially be used for achieving mode exchange between the two MGs without requiring wavelength conversion. This is similar to the parametric wavelength ex- change observed in single mode fibers [29]. To experimentally observe this, however, a high conversion efficiency is required. The reason is that when dealing with multiple modes, a crosstalk from one mode to another is observed at the output, which is introduced by the mode division multiplexer (MMUX), demultiplexer (MDMUX) and linear mode coupling in the HNL-FMF. Thus, when both the signal and idler are at the same frequency and the conversion efficiency is low, it would be difficult to differentiate between the generated idler in mode $b$ and the crosstalk of the original signal in mode $a$ to mode $b$. Unfortunately, due to the large insertion losses (ILs) of the MMUX/MDMUX used in this work, the conversion efficiency of our system is not high enough to observe mode exchange. Nevertheless, we believe by using other suitable MMUX/MDMUX systems such as photonic lanterns which have much smaller ILs, this would be possible.

\section{FIBER CHARACTERIZATION}

Even though the simulations in the previous section provide an estimate of the dispersion and nonlinear properties of the fabricated HNL-FMF, exact characterization of the HNL-FMF is required in order to perform the FWM experiments. Thus, we have characterized the fabricated fiber to find the linear and nonlinear properties of the supported eigenmodes, namely their attenuation coefficient $\alpha_{d B}$, relative inverse group velocity $\Delta \beta_{1}$, group velocity dispersion $\beta_{2}$, and nonlinear coefficient $\gamma$.

We use two liquid crystal on silicon (LCoS) based spatial light modulators (SLMs) as the MMUX and MDMUX [30, 31]. As shown in Fig. 3, by using a set of polarization diversity optics, the incoming beam from a single-mode fiber (SMF) is split to two 


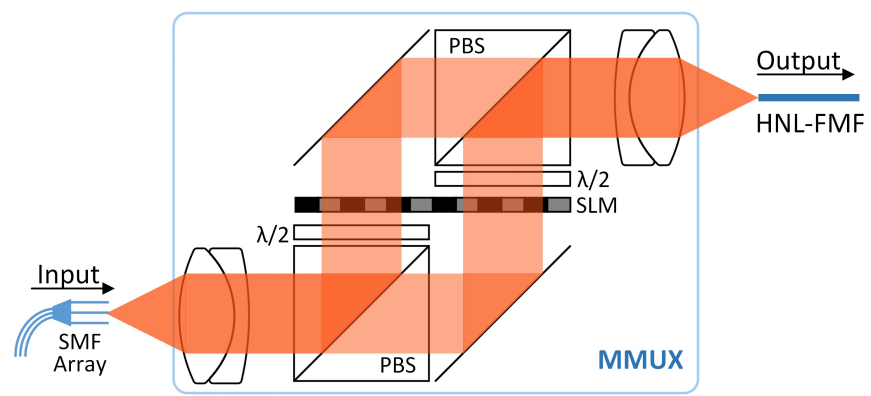

Fig. 3. SLM apparatus when used as MMUX for mode excitation of the fabricated HNL-FMF. PBS: polarizing beam splitter, $\lambda / 2$ : half-wave plate.

orthogonal polarizations. Each polarization is directed to a different area on the LCoS surface, making it possible to modulate the light in each polarization separately by displaying individual phase patterns on the area corresponding to each polarization. Another set of polarization diversity optics is used to recombine the two polarizations and focus the mode-multiplexed light into the HNL-FMF. The same configuration but in the reverse direction, is used as the MDMUX at the output of the HNL-FMF, where an arbitrary spatial state with any polarization can be converted back into a Gaussian beam. For exciting the eigenmodes within each MG, phase patterns of linearly polarized (LP) modes are displayed on the SLM. Each eigenmode in MG2 can be generated by combining different LP modes in orthogonal polarizations. For example, the phase pattern of $\mathrm{LP}_{11 \mathrm{~b}}$ in $\mathrm{x}$-polarization and that of $\mathrm{LP}_{11 \mathrm{a}}$ in y-polarization is used to generate $\mathrm{TE}_{01}$ mode (see Fig. 2 of Refs. [32, 33] for more details). Note that the polarization state of the input beam should be $45^{\circ}$ linearly polarized before the MMUX, in order to have equal power in each polarization for the excitation of the eigenmodes in MG2. After properly aligning the system, the mode extinction ratio (power ratio at the MDMUX between the excited mode and all other modes) is more than $8 \mathrm{~dB}$ for both MG1 and MG2.

For inter-modal FWM, since several input beams (pumps and signal) with different modes and wavelengths are interacting, an SMF array is used to combine these beams at the input of the MMUX. The fundamental mode in each input SMF can be individually converted to a desired mode of the HNL-FMF. The IL of the MMUX/MDMUX depends on the phase pattern displayed on the SLM and the number of ports used in the input SMF arrays.

\section{A. Attenuation Coefficient}

The attenuation coefficient of each MG is measured by using an optical time-domain reflectometer (OTDR). By changing the phase patterns displayed on the MMUX, we can selectively excite an MG and measure its attenuation. Since the modes within one MG couple linearly, we measure the losses of each MG instead of the individual modes. An optical pulse at $1550 \mathrm{~nm}$, with a width of $100 \mathrm{~ns}$, is generated by an OTDR. After propagating through a $300 \mathrm{~m} \mathrm{SMF}$, it travels through the MMUX, where it is converted to a specific MG. The pulse in the desired MG then propagates through the 2-km HNL-FMF and is converted back to the fundamental mode after the MDMUX. The back-scattered power in each MG is measured by the OTDR and shown in Fig. 4, as a function of the propagation distance. The first $300 \mathrm{~m}$ relatively flat stage corresponds to the SMF before the MMUX, while the subsequent stage from $300 \mathrm{~m}$ to $2300 \mathrm{~m}$ corresponds

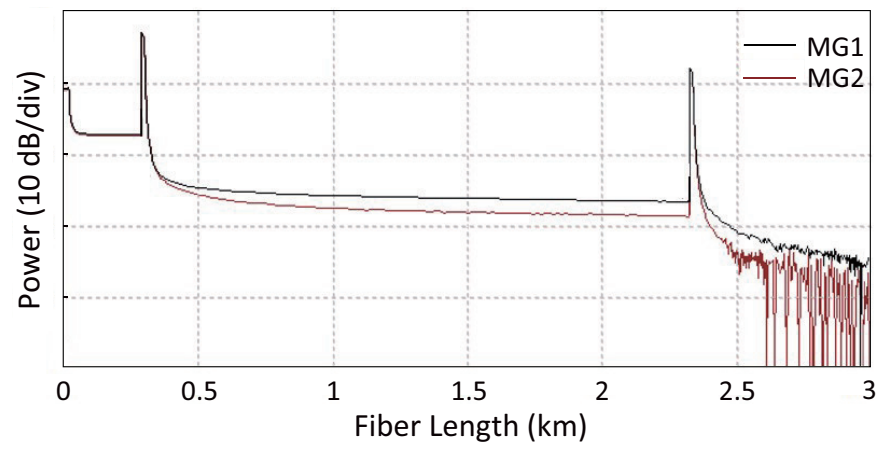

Fig. 4. Measured OTDR curves of both MGs.

to the 2-km HNL-FMF under test. The two peaks in each curve correspond to the Fresnel reflection of the fiber ends in the SLMs of the MMUX and MDMUX. The Fresnel reflection creates blindzones right after the peak locations, due to the high reflected power. Thus, for better accuracy, the data measured far away from the Fresnel reflections should be considered for the loss calculations; i.e. the data located in the linear regime around $1.3 \mathrm{~km}$ to $2.3 \mathrm{~km}$. By fitting a linear curve to these measurements, we obtain attenuation coefficients of 0.59 and $0.65 \mathrm{~dB} / \mathrm{km}$ for the MG1 and MG2, respectively. Since our HNL-FMF is the sample fabricated with traditional standard single mode fiber (SSMF) process, low loss characteristic is possible if both doping and drawing scheme are further optimized.

The SMF before the MMUX, helps us measure the IL of the MMUX for each MG. By fitting a linear curve to the SMF stage and comparing its power level to that of the HNL-FMF stage at $300 \mathrm{~m}$ (where the MMUX is located), one can find the IL of the MMUX, which is $8.3 \mathrm{~dB}$ when exciting MG1 and $10 \mathrm{~dB}$ for MG2. However, due to the relatively large insertion loss of the used MMUX, the dead zone after the reflection point of MMUX is relatively large. The selected data for the purpose of the linear fitting can cause an error on the injected power of around 0.3 $\mathrm{dB}$. The MMUX loss is required for calculating the input power to the FMF, which is particularly useful for characterizing the nonlinear coefficient of the HNL-FMF, as will be explained in Section 4-C.

\section{B. Group delay}

As mentioned in Section 2, the phase matching in inter-modal FWM processes strongly depends on the $\beta_{1}$ of the interacting modes. In this section, we characterize $\Delta \beta_{1}$ by measuring the differential mode group delay (DMGD) of the HNL-FMF. We perform this measurement based on the time-of-flight technique [34], using the setup shown in Fig. 5. Light coming from a tunable continuous-wave (CW) laser is modulated into pulse signals by a Mach-Zehnder intensity modulator (IM) with a pulsewidth of $100 \mathrm{ps}$ and a repetition rate of $100 \mathrm{MHz}$ generated by an arbitrary waveform generator (AWG). The modulated signal is adjusted by a polarization controller, then amplified by an erbium-doped fiber amplifier (EDFA) and launched into the HNL-FMF. The pulse signals propagating in different modes of the HNL-FMF will experience different time delays, therefore, after the MDMUX, they arrive at the photodiode (PD) at different times. By tuning the laser and measuring the relative time delay between the different modes, we can calculate $\Delta \beta_{1}$ at different wavelengths. Moreover, the wavelength dependence of the DMGD can be used to find the group velocity dispersion 


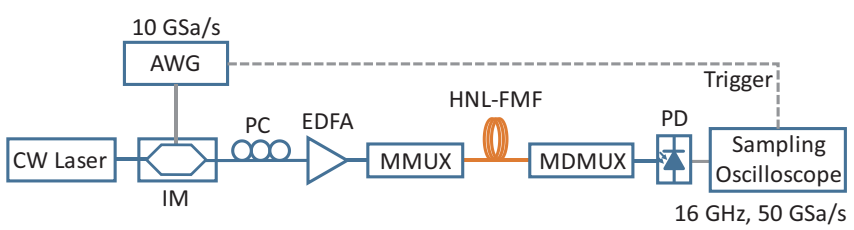

Fig. 5. The setup of time domain pulse response measurement.

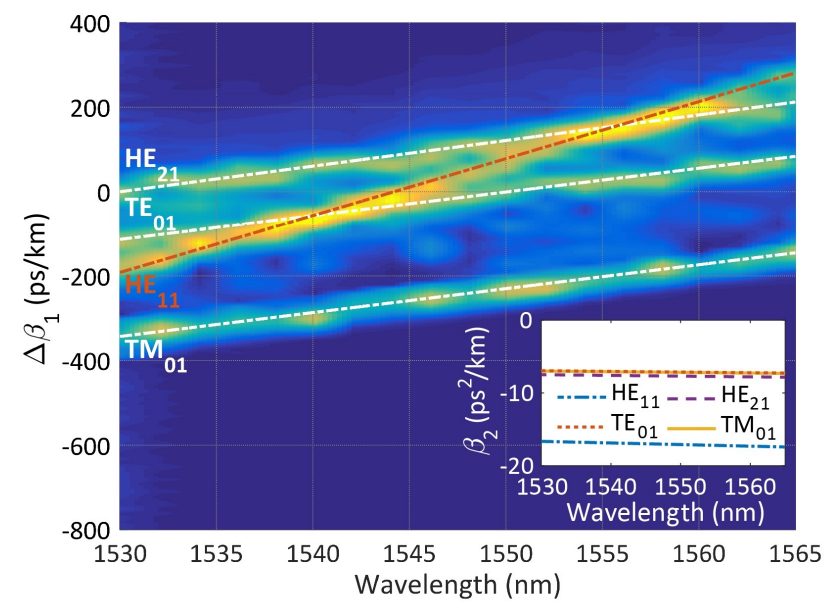

Fig. 6. Spectrogram of fiber pulse response over the C-band; inset: $\beta_{2}$ of each eigenmode over the C-band.

$\beta_{2}$ of each mode by

$$
\beta_{2}=-\frac{\lambda^{2}}{2 \pi c} \frac{d \beta_{1}}{d \lambda} .
$$

The signal wavelength is swept over the C-band, and the pulse response and DMGD is measured for different wavelengths. The measurements are normalized and plotted in the spectrogram, as shown in Fig. 6. Four approximately linear curves are clearly seen in this figure, of which the three parallel curves from top to bottom correspond to the three eigenmodes $\mathrm{HE}_{21}, \mathrm{TE}_{01}$ and $\mathrm{TM}_{01}$ in $\mathrm{MG}$, respectively, and the other curve corresponds to the $\mathrm{HE}_{11}$ mode. It can be observed that as predicted by simulations, the eigenmodes in MG2 are not degenerate and thus propagate through the HNL-FMF with different velocities. For any given wavelength, the vertical color distribution can be treated as the pulse response. Among the three parallel curves, there are some inhomogeneous areas colored in light blue, corresponding to the mode coupling between the eigenmodes in MG2. We can extract $\Delta \beta_{1}$ between any two modes and $\beta_{2}$ of each mode, at any wavelength over the $C$-band, from this figure. The extracted $\beta_{2}$ of each mode is shown in the inset of Fig. 6. Like other FMFs for inter-modal FWM [13, 27], the fabricated HNL-FMF is in the anomalous dispersion regime over the C-band.

It can be observed that the slopes of the $\Delta \beta_{1}$ curves for all guided modes in Fig. 6 are close to the simulations in Fig. 2(b), and the $\Delta \beta_{1}$ between any two eigenmodes in MG2 also generally agrees with simulations. However, the $\Delta \beta_{1}$ between $\mathrm{HE}_{11}$ mode in MG1 and any of the eigenmodes in MG2 has a positive shift of $\sim 200 \mathrm{ps} / \mathrm{km}$ in comparison with the simulations. As shown in Fig. 2(c), even 1\% variations in the fiber core radius $R$ or $\alpha$ parameter during the fabrication process leads to a shift of more than $200 \mathrm{ps} / \mathrm{km}$ in the $\Delta \beta_{1}$ between the MG1 and MG2, while it has a much less effect on $\Delta \beta_{1}$ within MG2. In addition to fabrication imperfections, some inhomogeneous stress was imposed on the HNL-FMF during the fiber spooling, which could have caused micro deformation of the fiber. The stress slightly reduces the core radius, and introduces a positive shift on the $\Delta \beta_{1}$ between MGs according to Fig. 2(c), which agrees with our measured result. There is also a relative shift of $\Delta \beta_{1}$ between arbitrary two of the eigenmodes in MG2, compared to Fig. 2(b). We infer that it is due to the circular asymmetry of the core induced by the random longitudinal fluctuations such as core non-circularity and the azimuthally inhomogeneous doping, as well as the macro bending during the fiber spooling [35].

The HNL-FMF presented here has some specific dispersion properties, making it suitable for various applications. First, the non-degenerate modes in MG2 give rise to three $\Delta \beta_{1}$ curves. Thus, a signal at any given wavelength in MG1 can be phasematched with three different modes in MG2 at three different wavelengths. The number of simultaneous phase-matched processes could be three times that of a commercial 2-MG FMF that has degenerate modes in MG2 [8]. Such property may be potential for the wavelength multicasting in mode division multiplexed (MDM) transmission, because compared to the schemes of using FMFs with more guided MGs [13], our HNL-FMF not only has smaller $A_{\text {eff }}$ leading to larger $\gamma$, but also has more balanced parametric gain properties of all mode channels in MG2 to mitigate the mode dependent gain/loss. Second, even though there is linear mode coupling within MG2, the different dispersion properties of the eigenmodes allow selective eigenmode amplification in MG2 through inter-modal FWM. This is possible since only the power in the phase-matched eigenmode will be amplified. Third, the three $\Delta \beta_{1}$ curves in MG2 are almost parallel over the C-band, indicating that the inter-modal BS processes between the eigenmodes in MG2 can be achieved with a relatively large bandwidth [10], which is from $7.7 \mathrm{~nm}$ to $18.7 \mathrm{~nm}$ in numerical simulations (considering a 10-dB bandwidth) depending on the selection of two interacting eigenmodes, leading to the potential application of broad-band mode and wavelength conversion. Finally, the $\mathrm{HE}_{11}$ curve has intersections with the $\mathrm{HE}_{21}$ and $\mathrm{TE}_{01}$ curves in the C-band. Hence, mode exchange becomes possible if the pumps and signal are placed near any of the crossing areas [29].

\section{Nonlinear Coefficient}

In order to measure the nonlinear coefficient $\gamma$ and nonlinear refractive index $n_{2}$ for both MGs, we send two pumps with a small wavelength difference to the designated MG and measure the power of the idlers generated by cascaded FWM processes [36]. The measurement setup is presented in Fig. 7. Optical signals coming from two continuous wave $(\mathrm{CW})$ lasers, operating around $1550 \mathrm{~nm}$ with a wavelength difference of $0.2 \mathrm{~nm}$, are aligned in polarization and combined by a $50: 50$ polarization maintaining (PM) coupler. After optical amplification, the sig-

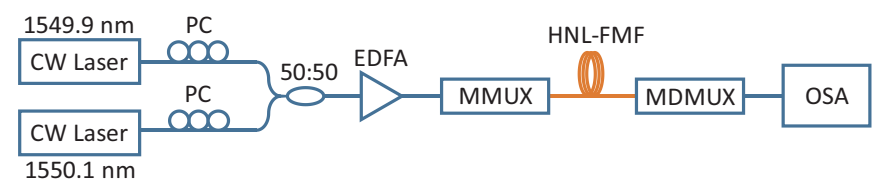

Fig. 7. Setup of CW-SPM nonlinear coefficient measurement. 


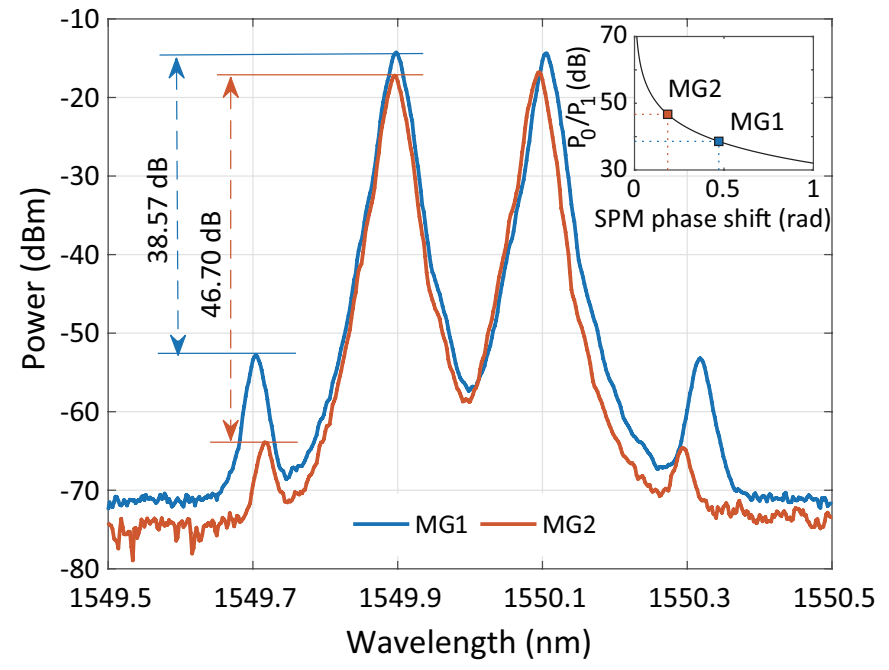

Fig. 8. SPM spectra of the beating signal after propagation in different MGs; inset: relationship between the SPM phase shift and the power ratio of the zero- and first- order harmonics, according to Ref. [36]. The two markers in the inset corresponds to the power ratios of each MG labelled out in the main figure.

nals are launched into a specific MG of the HNL-FMF. Optical interference occurs between the two optical waves, so the optical signal oscillates at the beat frequency of $25 \mathrm{GHz}$. If we treat the two pumps as a beat signal, the interaction process can be approximated as a self-phase modulation (SPM)-like process, because the wavelength difference of the pumps is small enough to neglect chromatic dispersion. As the beat signal propagates through the fiber, it experiences a nonlinear phase shift, which can be treated as a result of SPM determined by $\varphi_{\mathrm{SPM}}=2 \gamma L_{\mathrm{eff}} \bar{P}$, where $L_{\text {eff }}$ is the effective length of the fiber under test, and $\bar{P}$ is the average input power of both two wavelengths. The signal is then demultiplexed and observed by an optical spectrum analyzer (OSA) with a wavelength resolution of $0.01 \mathrm{~nm}$. The optical spectrum displays peaks at multiplies of the beat frequency if the nonlinear phase shift is large enough [24]. By measuring the power ratio between the peaks, it is possible to find $\varphi_{\mathrm{SPM}}$ and deduce the values of $\gamma$ and $n_{2}$ [36]. The zero- and first-order harmonics of each MG are displayed in Fig. 8, from which we can obtain the nonlinear phase shift of the two MGs, as shown in the inset of Fig. 8.

According to the IL measured in Section $4-\mathrm{A}, \bar{P}$ is calculated and consequently $\gamma$ for each individual MG is determined. The nonlinear coefficient of MG1 and MG2 is 5.7 and 2.8 $(\mathrm{W} \cdot \mathrm{km})^{-1}$, respectively, indicating a nonlinear refractive index $n_{2}$ of $2.98 \times 10^{-16} \mathrm{~cm}^{2} / \mathrm{W}$. The measured results are close to the $n_{2}=3.17 \times 10^{-16} \mathrm{~cm}^{2} / \mathrm{W}$, obtained from the analytical expression given in [28]. The eigenmodes in MG2 have very similar spatial distributions, so it is reasonable to consider a single $\gamma$ value for all modes of MG2. The inter-modal $\gamma$ is estimated to be $2.8(\mathrm{~W} \cdot \mathrm{km})^{-1}$, based on the measured $n_{2}$ and the estimated inter-modal $A_{\text {eff }}$.

\section{INTER-MODAL FOUR-WAVE MIXING}

Based on the characterized parameters in the previous sections, we conducted an experiment to achieve simultaneous intermodal FWM among multiple eigenmodes of the HNL-FMF. The experimental setup is shown in Fig 9. Pump 1 and Signal are in the $\mathrm{HE}_{11}$ mode, at $1550 \mathrm{~nm}$ and $1549.6 \mathrm{~nm}$, respectively. Pump 2 is launched into the $\mathrm{HE}_{21}$ mode at $1542.3 \mathrm{~nm}$, and Pump 3 in the $\mathrm{TE}_{01}$ mode is at $1565 \mathrm{~nm}$. The wavelengths of the interacting waves are chosen based on the group delay properties of the modes, as shown in Fig. 6, so that the inter-modal FWM processes are phase-matched. The polarization controllers right after the Pump 1 and Signal lasers are used to align the polarization of the two beam. A polarization-maintaining 90:10 coupler is used to couple Pump 1 and Signal into a high-gain EDFA, whose output power is set to be $28.5 \mathrm{dBm}$. A thin-film tunable optical bandpass filter (TBPF) with a bandwidth of 1-nm is used after the EDFA to filter out the amplified spontaneous emission (ASE) noise. For Pump 2 and Pump 3, the bandwidths of the TBPFs are chosen to be $0.6-\mathrm{nm}$ to ensure a good optical signal-to-noise ratio (OSNR) around each idler wavelength. Note that the polarization states of Pump 2 and Pump 3 before the MMUX should be linearly polarized with an azimuth of $45^{\circ}$, as described in Section 4. However, Pump 1 does not need to be co-polarized with Pump 2 and Pump 3, because $\mathrm{HE}_{21}$ and $\mathrm{TM}_{01}$ modes are vector eigenmodes with variable polarization state at arbitrary spacial location, as shown in the inset of Fig. 2(a). For an arbitrary azimuth of the linearly polarized $\mathrm{HE}_{11}$ mode, the co-polarized component and cross-polarized component of the inter-modal FWM exist side by side, and the total efficiency is always the same due to the symmetric spatial evolution of the polarization states. After aligning the polarization states of all the input beams by three polarization controllers, they are combined together using a SMF array and launched into the MMUX, where each beam is converted to the desired eigenmode. After few-mode transmission over the 2-km HNL-FMF, mode division demultiplexing is accomplished and the output spectrum is observed by an OSA.

It is noteworthy that there is a large loss in each mode channel, between the EDFA output power and the power coupled into the corresponding mode in the HNL-FMF. The loss consists of ILs of all the connectors, the TBPF and mainly the MMUX. By implementing the OTDR measurements, we obtained total losses of $10.7 \mathrm{~dB}, 12.8 \mathrm{~dB}$ and $12.6 \mathrm{~dB}$ for the $\mathrm{HE}_{11}, \mathrm{HE}_{21}$ and $\mathrm{TE}_{01}$ modes, respectively. Thus, the actual power of Pump 1 injected into the $\mathrm{HE}_{11}$ mode at the HNL-FMF input is only $17.3 \mathrm{dBm}$, while the pump powers of $\mathrm{HE}_{21}$ mode and $\mathrm{TE}_{01}$ mode are 6.2 $\mathrm{dBm}$ and $15.9 \mathrm{dBm}$, respectively, since the EDFA output power for the $\mathrm{HE}_{21}$ mode is only $19 \mathrm{dBm}$. Under such power level of less than $100 \mathrm{~mW}$, no geometric parametric instability (GPI) effect can be stimulated over the 2-km HNL-FMF. Furthermore, the higher-order dispersion can be neglected in the analysis for

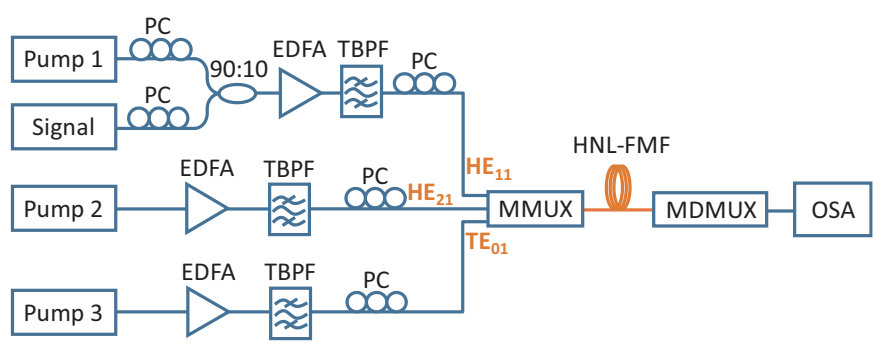

Fig. 9. Experimental setup for inter-modal FWM. Signal and Pump 1 are connected with the $\mathrm{HE}_{11}$ port of the MMUX, while Pump 2 and Pump 3 are connected with the $\mathrm{HE}_{21}$ port and $\mathrm{TE}_{01}$ port, respectively. 


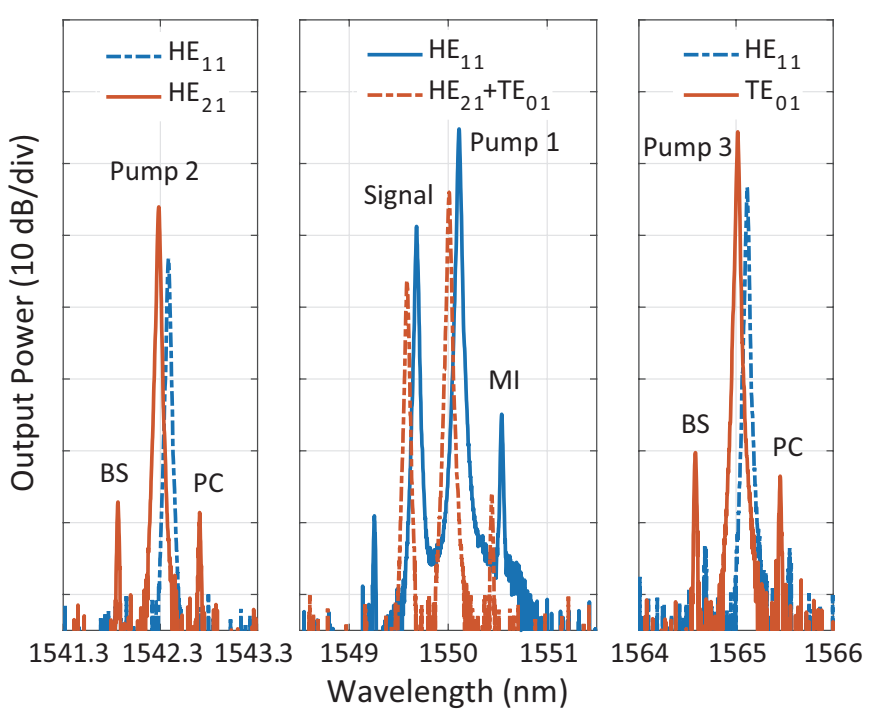

Fig. 10. Output spectra after mode demultiplexing. Two different MGs are received separately by changing the corresponding phase pattern in the MDMUX. For easier observation, a red-shift of $0.1 \mathrm{~nm}$ is intentionally added on the spectrum when receiving $\mathrm{HE}_{11}$ mode. Dashed lines mean the crosstalk from one MG to the other.

the inter-modal FWM experiment [22]. The Raman noise can also be neglected by considering the pump power and the small wavelength detuning between Signal and Pump 1 [37].

Figure 10 shows the output spectra in each MG. The spectrum of the output $\mathrm{HE}_{11}$ mode is intentionally red-shifted by $0.1 \mathrm{~nm}$ for ease of observation, so that the spectra related to the two MGs do not overlap. For wavelengths near $1550 \mathrm{~nm}$, we can clearly see intra-modal MI idlers at $1550.4 \mathrm{~nm}$ and $1549.2 \mathrm{~nm}$ in the $\mathrm{HE}_{11}$ mode. The crosstalk to MG2 (shown in red) shows a mode extinction ratio of at least $8 \mathrm{~dB}$. For TE 01 mode at $1565 \mathrm{~nm}$, the wavelength conversion efficiency (CE), which is defined as the power ratio between the output idler and the output Signal, is $-31.5 \mathrm{~dB}$ and $-34.8 \mathrm{~dB}$ for the BS and PC processes, respectively. For $\mathrm{HE}_{21}$ mode with a lower input power, $\mathrm{CE}$ is $-38.4 \mathrm{~dB}$ and $-39.8 \mathrm{~dB}$ for BS and PC, respectively. The generated idler power is proportional to the input pump power and therefore the $\mathrm{CE}$ in the $\mathrm{TE}_{01}$ mode is higher than that of the $\mathrm{HE}_{21}$ mode. Due to the existence of random birefringence fluctuations, the output idler power in each eigenmode in MG2 is reduced [38]. Moreover, there is also crosstalk among the eigenmodes in MG2 due to the mode coupling. However, the crosstalk of pump from one specific eigenmode to another eigenmode is not phase-matched with respect to the Pump 1 and Signal. Therefore, no idlers due to the crosstalk will be generated, and consequently the idler power coupled into other eigenmodes will not be amplified. Moreover, the mode coupling within MG2 only causes the reduction of the idler power in the designated eigenmode and the deterioration of CE. Furthermore, we observe that all the idlers have power fluctuations within $5 \mathrm{~dB}$, which is also due to the random mode coupling among the involved modes. Such random reduction of the idler power in the designated eigenmode is the main limitation of using the non-degenerate eigenmodes. In addition, small mechanical vibration or thermal variation can affect the value of $\Delta \beta_{1}$ and consequently lead to

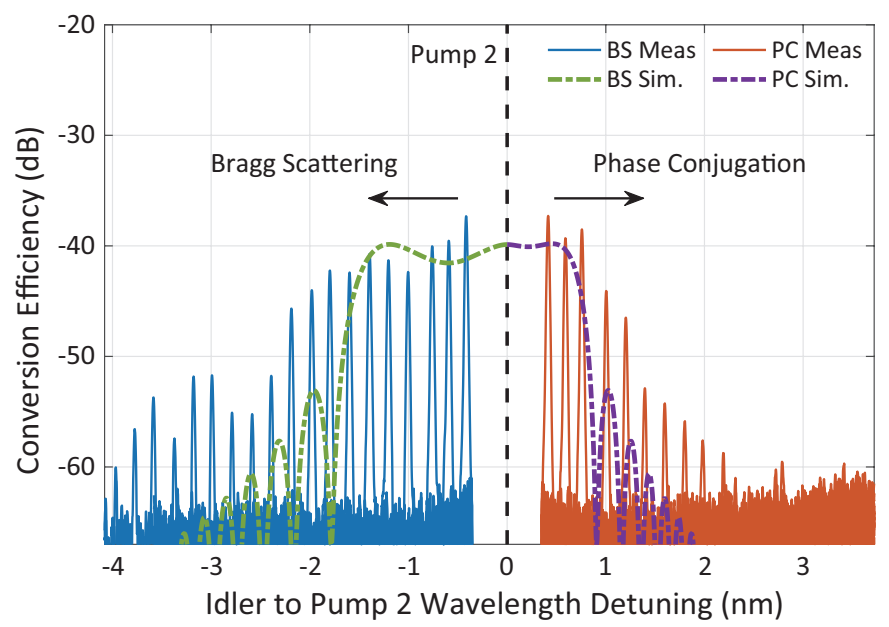

Fig. 11. Spectra of conversion efficiency when the Signal wavelength varies from $1549.6 \mathrm{~nm}$ to $1546 \mathrm{~nm}$. The blue curves correspond to the $\mathrm{BS}$ idlers, while the orange curves correspond to the PC idlers. The dashed-dotted curves correspond to the CE curves found through simulations, and the black dashed line shows where the Pump 2 is located.

the power fluctuations of the idlers [39]. Nevertheless, higher CE can be achieved if MMUX/MDMUX devices with smaller ILs (such as photonic lanterns) is used, or using higher power EDFAs together with stimulated Brillouin scattering (SBS) suppression scheme. Using a longer HNL-FMF is also helpful for the CE improvement. Furthermore, if the Signal carries data, both BS and PC idlers of each eigenmode will carry the data (or phase conjugated data). The $\mathrm{TM}_{01}$ mode could also be used as another mode channel to perform inter-modal FWM over the L-band, as can be deduced from Fig. 6. Thus, this HNL-FMF has the potential to achieve 1 to 6 inter-modal wavelength multicasting within only two MGs.

By changing the Signal wavelength, the bandwidths of BS and $\mathrm{PC}$ between $\mathrm{HE}_{11}$ and $\mathrm{HE}_{21}$ modes are invesgated in Fig. 11. The Signal to Pump 1 wavelength detuning is varied from 0.4 $\mathrm{nm}$ to $4 \mathrm{~nm}$, with a $0.2 \mathrm{~nm}$ interval. As shown in Fig. 11, the blue peaks left to the Pump 2 wavelength correspond to the idlers of BS, while the red peaks, right to Pump 2, are the idlers of PC. Taking a 10-dB bandwidth into consideration, the bandwidths of BS and PC processes are $2.2 \mathrm{~nm}$ and $1.2 \mathrm{~nm}$, respectively. The bandwidth of BS is larger than PC, in agreement with theoretical predictions [10]. We also simulated these nonlinear processes by numerically solving the coupled nonlinear Schrödinger equations that describe the propagation of the eigenmodes in the HNL-FMF [7]. The simulated bandwidths for the BS and PC process are $1.7 \mathrm{~nm}$ and $0.8 \mathrm{~nm}$, respectively, as shown in Fig. 11. The bandwidth difference between simulation and experiment may be due to the fluctuations of $\Delta \beta_{1}$ along the HNL-FMF induced by the random local perturbations, especially for our HNL-FMF with a small core radius [38]. There is a trade-off between the fiber length and the bandwidth of the inter-modal processes. Given the SLM losses, we needed a long fiber length to observe the inter-modal nonlinearities. However, by using other MMUX/MDMUX methods, it is possible to shorten the fiber and increase the bandwidth of the BS and PC interactions. 


\section{CONCLUSIONS}

Design, fabrication and characterization of an HNL-FMF is presented, with a record-high inter-modal nonlinear coefficient of $2.8(\mathrm{~W} \cdot \mathrm{km})^{-1}$. High germanium concentration in the core breaks the mode degeneracy in the second MG, and results in different dispersion properties for the eigenmodes within that MG. This allows multiple inter-modal interactions among the eigenmodes. The number of possible inter-modal FWM processes between the two MGs is 3 times that of a commercial two-MG FMF and in addition inter-modal FWM within MG2 can also be realized in the HNL-FMF presented here. Accordingly, simultaneous wavelength conversion among three eigenmodes is experimentally verified over the C-band, which shows the great potential of this HNL-FMF in all-optical signal processing applications, such as wavelength multicasting and eigenmode conversion. According to simulations, $\Delta \beta_{1}$ and consequently the phase matching of the inter-modal FWM processes between the MGs is sensitive to fabrication imperfections, which should be taken into account while fabricating such fibers.

\section{FUNDING.}

National Key R\&D Program of China (2018YFB1801002); National Natural Science Foundation of China (NSFC) (61711530043); Fundamental Research Funds for the Central Universities, HUST (2018JYCXJJ024); Swedish Research Council (VR) (2015-00535, 2017-05157); Swedish Foundation for International Cooperation in Higher Education (STINT) (CH2016-6754).

\section{REFERENCES}

1. D. J. Richardson, J. M. Fini, and L. E. Nelson, "Space-division multiplexing in optical fibres," Nat Photon 7, 354-362 (2013).

2. R. Ryf, S. Randel, A. H. Gnauck, C. Bolle, A. Sierra, S. Mumtaz, M. Esmaeelpour, E. C. Burrows, R. J. Essiambre, P. J. Winzer, D. W. Peckham, A. H. McCurdy, and R. Lingle, "Mode-division multiplexing over $96 \mathrm{~km}$ of few-mode fiber using coherent $6 \times 6$ mimo processing," J. Light. Technol. 30, 521-531 (2012).

3. C. Koebele, M. Salsi, L. Milord, R. Ryf, C. Bolle, P. Sillard, S. Bigo, and G. Charlet, " $40 \mathrm{~km}$ transmission of five mode division multiplexed data streams at $100 \mathrm{gb} / \mathrm{s}$ with low mimo-dsp complexity," in 37th European Conference and Exhibition on Optical Communication 2011, (2011), pp. 1-3.

4. R. Stolen, "Phase-matched-stimulated four-photon mixing in silica-fiber waveguides," IEEE J. Quantum Electron. 11, 100-103 (1975).

5. N. Zhao, B. Huang, R. Amezcua-Correa, X. Li, and G. Li, "Few-mode fiber optical parametric amplifier," in Optical Fiber Communication Conference/National Fiber Optic Engineers Conference 2013, (2013), p. OTu2D.5.

6. M. Guasoni, "Generalized modulational instability in multimode fibers: Wideband multimode parametric amplification," Phys. Rev. A 92, 033849 (2015).

7. E. Nazemosadat, A. Lorences-Riesgo, M. Karlsson, and P. A. Andrekson, "Design of highly nonlinear few-mode fiber for c-band optical parametric amplification," J. Light. Technol. 35, 2810-2817 (2017).

8. Y. Xiao, R.-J. Essiambre, M. Desgroseilliers, A. M. Tulino, R. Ryf, S. Mumtaz, and G. P. Agrawal, "Theory of intermodal four-wave mixing with random linear mode coupling in few-mode fibers," Opt. Express 22, 32039-32059 (2014).

9. R. J. Essiambre, M. A. Mestre, R. Ryf, A. H. Gnauck, R. W. Tkach, A. R. Chraplyvy, Y. Sun, X. Jiang, and R. Lingle, "Experimental investigation of inter-modal four-wave mixing in few-mode fibers," IEEE Photon. Technol. Lett. 25, 539-542 (2013).

10. S. M. M. Friis, I. Begleris, Y. Jung, K. Rottwitt, P. Petropoulos, D. J. Richardson, P. Horak, and F. Parmigiani, "Inter-modal four-wave mixing study in a two-mode fiber," Opt. Express 24, 30338-30349 (2016).
11. O. F. Anjum, P. Horak, Y. Jung, M. Suzuki, Y. Yamamoto, T. Hasegawa, P. Petropoulos, D. J. Richardson, and F. Parmigiani, "Bandwidth enhancement of inter-modal four wave mixing bragg scattering by means of dispersion engineering," APL Photonics 4, 022902 (2019).

12. J. Demas, L. Rishøj, X. Liu, G. Prabhakar, and S. Ramachandran, "Intermodal group-velocity engineering for broadband nonlinear optics," Photon. Res. 7, 1-7 (2019).

13. H. Zhang, M. Bigot-Astruc, L. Bigot, P. Sillard, and J. Fatome, "Multiple modal and wavelength conversion process of a 10-gbit/s signal in a 6-Ip-mode fiber," Opt. Express 27, 15413-15425 (2019).

14. G. Rademacher, R. Ryf, N. K. Fontaine, H. Chen, R. M. Jopson, R. Essiambre, B. J. Puttnam, R. S. Luís, Y. Awaji, N. Wada, S. Gross, N. Riesen, M. Withford, Y. Sun, and R. Lingle, "Experimental investigation of parametric mode and wavelength conversion in a $4.7 \mathrm{~km}$ few-mode fiber," in 2018 European Conference on Optical Communication (ECOC), (2018), pp. 1-3.

15. E. Nazemosadat and A. Mafi, "Design considerations for multicore optical fibers in nonlinear switching and mode-locking applications," J. Opt. Soc. Am. B 31, 1874-1878 (2014).

16. G. Lopez-Galmiche, Z. S. Eznaveh, M. A. Eftekhar, J. A. Lopez, L. G. Wright, F. Wise, D. Christodoulides, and R. A. Correa, "Visible supercontinuum generation in a graded index multimode fiber pumped at 1064 nm," Opt. Lett. 41, 2553-2556 (2016).

17. L. G. Wright, D. N. Christodoulides, and F. W. Wise, "Controllable spatiotemporal nonlinear effects in multimode fibres," Nat Photon $\mathbf{9}$, 306-310 (2015).

18. H. Pourbeyram, E. Nazemosadat, and A. Mafi, "Detailed investigation of intermodal four-wave mixing in smf-28: blue-red generation from green," Opt. Express 23, 14487-14500 (2015).

19. J. Cheng, M. E. V. Pedersen, K. Charan, K. Wang, C. Xu, L. GrünerNielsen, and D. Jakobsen, "Intermodal four-wave mixing in a higherorder-mode fiber," Appl. Phys. Lett. 101, 161106 (2012).

20. E. Nazemosadat, H. Pourbeyram, and A. Mafi, "Phase matching for spontaneous frequency conversion via four-wave mixing in gradedindex multimode optical fibers," J. Opt. Soc. Am. B 33, 144-150 (2016).

21. R. Dupiol, A. Bendahmane, K. Krupa, J. Fatome, A. Tonello, M. Fabert, V. Couderc, S. Wabnitz, and G. Millot, "Intermodal modulational instability in graded-index multimode optical fibers," Opt. Lett. 42, 3419-3422 (2017).

22. A. Bendahmane, K. Krupa, A. Tonello, D. Modotto, T. Sylvestre, V. Couderc, S. Wabnitz, and G. Millot, "Seeded intermodal four-wave mixing in a highly multimode fiber," J. Opt. Soc. Am. B 35, 295-301 (2018).

23. K. Inoue, "Four-wave mixing in an optical fiber in the zero-dispersion wavelength region," J. Light. Technol. 10, 1553-1561 (1992).

24. G. P. Agrawal, "Nonlinear fiber optics, 4th ed." (Academic Press, San Diego, 2007).

25. R. H. Stolen, J. E. Bjorkholm, and A. Ashkin, "Phase-matched threewave mixing in silica fiber optical waveguides," Appl. Phys. Lett. 24, 308-310 (1974).

26. C. J. McKinstrie, S. Radic, and M. G. Raymer, "Quantum noise properties of parametric amplifiers driven by two pump waves," Opt. Express 12, 5037-5066 (2004).

27. G. Rademacher, R. S. Luís, B. J. Puttnam, Y. Awaji, M. Suzuki, T. Hasegawa, and N. Wada, "Wide-band intermodal wavelength conversion in a dispersion engineered highly nonlinear fmf," in Optical Fiber Communication Conference (OFC) 2019, (Optical Society of America, 2019), p. W1C.4.

28. K. Nakajima and M. Ohashi, "Dopant dependence of effective nonlinear refractive index in geo2- and f-doped core single-mode fibers," IEEE Photonics Technol. Lett. 14, 492-494 (2002).

29. K. Uesaka, K. K. Y. Wong, M. E. Marhic, and L. G. Kazovsky, "Wavelength exchange in a highly nonlinear dispersion-shifted fiber: theory and experiments," IEEE J. Sel. Top. Quantum Electron. 8, 560-568 (2002).

30. J. Carpenter, B. C. Thomsen, and T. D. Wilkinson, "Degenerate modegroup division multiplexing," J. Light. Technol. 30, 3946-3952 (2012).

31. J. Carpenter, B. J. Eggleton, and J. Schröder, "110x110 optical mode transfer matrix inversion," Opt. Express 22, 96-101 (2014). 
32. Q. Zhan, "Cylindrical vector beams: from mathematical concepts to applications," Adv. Opt. Photon. 1, 1-57 (2009).

33. Y. Yang, J. Cui, S. Fu, M. Tang, and D. Liu, "All-fiber flexible generation of the generalized cylindrical vector beam (cvb) over the c-band," IEEE J. Sel. Top. Quantum Electron. 26, 1-7 (2020).

34. J. Cheng, M. E. V. Pedersen, K. Wang, C. Xu, L. Grüner-Nielsen, and D. Jakobsen, "Time-domain multimode dispersion measurement in a higher-order-mode fiber," Opt. Lett. 37, 347-349 (2012).

35. J. Su, X. Dong, and C. Lu, "Characteristics of few mode fiber under bending," IEEE J. Sel. Top. Quantum Electron. 22, 139-145 (2016).

36. A. Boskovic, S. V. Chernikov, J. R. Taylor, L. Gruner-Nielsen, and O. A. Levring, "Direct continuous-wave measurement of $\mathrm{n} 2$ in various types of telecommunication fiber at $1.55 \mu \mathrm{m}$," Opt. Lett. 21, 1966-1968 (1996).

37. K. Krupa, A. Tonello, V. V. Kozlov, V. Couderc, P. D. Bin, S. Wabnitz, A. Barthélémy, L. Labonté, and S. Tanzilli, "Bragg-scattering conversion at telecom wavelengths towards the photon counting regime," Opt. Express 20, 27220-27225 (2012).

38. M. Guasoni, F. Parmigiani, P. Horak, J. Fatome, and D. J. Richardson, "Intermodal four-wave mixing and parametric amplification in kilometerlong multimode fibers," J. Light. Technol. 35, 5296-5305 (2017).

39. M. Esmaeelpour, R. Essiambre, N. K. Fontaine, R. Ryf, J. Toulouse, Y. Sun, and R. Lingle, "Power fluctuations of intermodal four-wave mixing in few-mode fibers," J. Light. Technol. 35, 2429-2435 (2017). 